\title{
EXPERIENCIAS ESTÉTICAS DE EMPODERAMIENTO EN NIÑOS, JÓVENES Y ADULTOS DEL CENTRO DE EXPRESIÓN CULTURAL DE USME
}

\section{AESTHETIC EXPERIENCES OF EMPOWERMENT IN CHILDREN, YOUTH AND ADULTS OF THE}

CENTER OF CULTURAL EXPRESSION OF USME

\section{EXPERIÊNCIAS ESTÉTICAS DE EMPODERAMENTO EM CRIANÇAS, JOVENS E ADULTOS DO} CENTRO DE EXPRESSÃO CULTURAL DE USME

Laura Nataly García Flórez reloj56@yahoo.com Universidad Distrital Francisco José de Caldas Bogotá - Colombia

Diagramación

Sindy Catherine Charcas Ibarra

Fotografia de Portada Santiago Ardila Acero

Encuentre este artículo en

https://revistas.uniminuto.edu/index.php/IYD/index

Para citar este artículo / To cite this article

García-Flórez, L. (2020). Experiencias estéticas de empoderamiento en niños, jóvenes y adultos del Centro de

Expresión Cultural de Usme. Inclusión \& Desarrollo, 7 (I), pp 2I -29
Fecha de recepción: 2 octubre de 2019 Fecha de aceptación: 2 de noviembre 2019 Fecha de publicación: 31 de diciembre de 2019 


\section{RESUMEN}

Este artículo es el resultado de un ejercicio de sistematización que muestra la experiencia de un proyecto artístico llevado a cabo en el barrio Santa Librada en localidad quinta de Usme que durante más de treinta años ha posicionado su propuesta transformadora en la comunidad garantizando la permanencia de núcleos familiares que por tres generaciones se han involucrado en el proyecto, haciendo de este un lugar de múltiples aprendizajes en donde los sujetos construyen su propia historia desde el arte como eje central para la reivindicación y posicionamiento de niños y jóvenes líderes de su propia comunidad. Esta sistematización recoge las voces de tres familias participantes que por más de diez años han creído en un proyecto alternativo para su sector. Ellos y muchas personas más enriquecen esta experiencia desde sus prácticas educativas, culturales y sociales que en los sectores populares son tan necesarias, sobre todo por el reconocimiento y fortalecimiento de una memoria colectiva que les permita escribir su historia.

Palabras claves: educación, empoderamiento, comunidad, sistematización, experiencia, participación.

\section{ABSTRACT}

This article is the result of a systematization exercise that shows the experience of an artistic project carried out in the Santa Librada neighborhood in the fifth town of Usme that has positioned its transformative proposal in the community for more than thirty years, guaranteeing the permanence of family nuclei that for three generations have been involved in the project, making this a place of multiple learning where subjects build their own history from art as a central axis for the defense and positioning of children and young leaders of their own community. This systematization gathers the voices of three participating families who for over ten years have believed in an alternative project for their sector. They and many more people enrich this experience from their educational, cultural and social practices that in the popular sectors are so necessary, especially for the recognition and strength of a collective memory that allows them to write their history.

Keywords: education, empowerment, community, systematization, experience, participation.

\section{SUMÁRIO}

Este artigo é resultado de um exercício de sistematização que mostra a experiência de um projeto artístico realizado no bairro de Santa Librada, em Usme, Bogotá, que posiciona sua proposta transformadora na comunidade há mais de trinta anos, garantindo a permanência de núcleos de parentes que por três gerações se envolveram no projeto, tornando este um local de aprendizado múltiplo, onde os sujeitos constroem sua própria história a partir da arte como o eixo central para a reivindicação e o posicionamento de crianças e jovens como lideranças de sua própria comunidade. Essa sistematização reúne as vozes de três famílias participantes que há mais de dez anos acreditam em um projeto alternativo para seu setor. Eles e muitas mais pessoas enriquecem essa experiência com suas práticas educacionais, culturais e sociais que nos setores populares são tão necessárias, especialmente para o reconhecimento e fortalecimento de uma memória coletiva que lhes permita escrever sua história.

Palavras-chave: educação, empoderamento, comunidade, sistematização, experiência, participação. 


\section{Introducción}

El Centro de Expresión Cultural Fe y Alegría de Santa Librada (CEC) ubicado en la localidad Quinta Usme nace en el año de 1988 en cabeza de las Hermanas Carmelitas de la caridad Vedruna, una comunidad de religiosas que empiezan a desarrollar tres líneas de acción en pro del fortalecimiento y progreso de la comunidad. Dichas líneas son: educación, salud y procesos comunitarios.

El impacto del proyecto en la localidad ha sido desde ese entonces muy positivo, ya que en años anteriores no existían escuelas o iniciativas artísticas de las cuales la población pudiera verse beneficiada. Un espacio donde los niños y jóvenes ocuparan sus tiempos libres, aprendieran un arte y se relacionaran con niños de su edad. Ahora, debido a su trayectoria, es un proyecto muy reconocido en el sector.

La hermana Pilar Alonso Soler, directora del CEC, fue quien impulsó esta iniciativa artística de formación integral que, a la par del arte, trabajaba otros aspectos complementarios: formación en valores desde talleres y cine foros, asambleas juveniles relacionadas con temas de formación política, talleres de liderazgo, entre otros. Estas experiencias integradoras hicieron que los niños y jóvenes de la época crecieran acompañados de un espacio donde conocieran gente contemporánea, aprendieran e intercambiaran su saber, pero, sobre todo, entendieran la importancia del trabajo comunitario como una razón fundamental en la construcción del proyecto.

En el año 2000, las Hermanas Vedruna se trasladaron a otro lugar de la ciudad, dejando el proyecto del CEC en manos de un grupo de jóvenes que se formaron en el espacio y que buscaron darle continuidad al legado que dejaron ellas. $A$ partir de ese año, y durante los catorce años siguientes, fueron varias personas las que pasaron por la dirección del centro.

En el año 2015, el proyecto cerró sus puertas debido a que los últimos recursos económicos se agotaron. Durante ese año, los jóvenes que llevaban varios años en el proceso se resistieron a la posibilidad de un cierre definitivo buscando alternativas para volver a reabrir el lugar. Es ahí donde aparece una docente de danzas que participó en el proceso del CEC desde su infancia, pasando por distintos espacios del proyecto. Actualmente es ella quien lidera el espacio atendiendo una población de 100 niños y jóvenes aproximadamente.

Esta experiencia artística, como muchas otras existentes en el país, permite identificar el arte como implementación de herramientas pedagógicas, para el desarrollo cognitivo, auditivo, psicomotor y visual de los niños y las niñas. De ahí la importancia de fortalecer procesos cognitivos, a través de prácticas de enseñanza y aprendizaje en las cuales se promueva el desarrollo de habilidades artísticas, pues como sabemos, en los escenarios de formación se vienen configurando las identidades juveniles, mediadas en muchos casos por la influencia que ejercen expresiones artísticas como las artes plásticas, las artes visuales, la literatura, la música, la danza, entre otras.

Por tal razón, interesa a este ejercicio de sistematización avanzar en una indagación por los procesos de empoderamiento de los niños y jóvenes en torno a los espacios de educación no formal, desde ejercicios prácticos que muestren sus percepciones frente al estar en un espacio y compartir con otros sujetos. Esto con el fin de evidenciar cómo suceden dichas prácticas y cómo, desde una propuesta concreta, se puede pensar una relación más cercana entre la cotidianidad de los niños y niñas y la manifestación social y cultural de su entorno.

Ahora bien, el arte no solo es para contemplar, sino que es un acto y como tal hace parte de una interacción y construcción colectiva que incide en la cultura de un lugar, ya que de alguna manera está influenciando las formas de pensar y percibir el mundo de los sujetos que allí se encuentran, se puede decir que espacios como los del Centro de Expresión Cultural están encaminados en la construcción de percepciones, buscando otras formas de ver y sentir, esto de la mano con la necesidad que tienen las personas de hacer parte de ámbitos y territorios por los que se va pasando a lo largo de la vida. La mayoría de niños y jóvenes buscan un lugar diferente al de la casa y escuela para conocer personas contemporáneas a su edad, relacionarse y convivir en la construcción de aprendizajes colectivos donde ellos aporten desde su saber.

\section{Estado del arte}

Para la composición del estado del arte, se exponen algunos trabajos que presentan la investigación reciente en cuanto a la categoría relacionadas con procesos comunitarios de participación social, que, en conjunción, sirven de referente para el desarrollo de la presente investigación.

\section{La participación como experiencia}

Al analizar algunos textos referentes al tema del presente proyecto, se puede evidenciar investigaciones relacionadas con procesos de participación y sistematización de experiencias de trabajo comunitario. A continuación, se describen, uno a uno, los documentos trabajados.

Para empezar, Alfaro y Sura (2007), en su tesis Teatro comunitario como proceso de transformación social realizan la sistematización de tres experiencias en Chile las cuales recopilan el trabajo comunitario que desde el teatro proponen una investigación frente a la construcción de conocimiento, sus lenguajes y formas de comunicación.

En un primer momento, hacen un análisis frente al concepto de identidad cultural a partir del análisis de procesos sociales como elaboración de proyectos políticos. Desde ahí parte el trabajo de cultura popular, el cual permite desarrollar "identidades" desde la construcción de conocimientos que parten de las experiencias comunitarias.

En un segundo momento, Alfaro y Sura (2007) cuentan cada una de las experiencias a sistematizar. En este acercamiento, se realiza una descripción que permite ubicar al lector frente a varias de las características de las experiencias, como, por ejemplo: cómo se fundó, bajo qué dinámica, con qué propósitos, entre otros. 
Como objetivo se proponen generar la reflexión de tres experiencias artísticas. La primera es La Sarunga Sureña, la cual fue desarrollada dentro de la localidad de Ercilla. La segunda es De Dudosa Procedencia de La Villa La Búsqueda y del trabajo Guiado por Teatro PASMI con las compañías "llusiones" y "Fénix" de CCP Colina I. La tercera es Sueños de Libertad de CDP San Miguel. Todas estas son llevadas a cabo en el año 2007, para después dar cuenta de procesos de participación y de identidades culturales dentro de sus propias comunidades.

Como resultados frente a este trabajo Teatro comunitario como proceso de transformación social se propone la sistematización de experiencias como una forma de realizar investigación, en donde se constituyen herramientas y conocimientos desde y para los sujetos. Como segundo aspecto, se concluye que toda acción constituye una práctica política y en un proceso critico de sistematización el análisis de la realidad debe dar cuenta de los conocimientos que se generan en la cotidianidad. Por último, las autoras refieren al teatro comunitario como un proceso transformador, ya que desde la participación se contiene en su totalidad la colaboración, cohesión y organización de sus participantes.

Ahora bien, por otro lado, Morales y Pineda (2017), en la tesis titulada Trinchera: Bosa, Arte, Saberes y Territorio: una alternativa agonística de hacer escuela, muestran una propuesta pedagógica a partir de la interacción entre las ciencias sociales y las artes. Se implementa una dinámica de trabajo que fomenta la promoción de la formación de subjetividades políticas de procesos de construcción y de devolución creativa del conocimiento a partir de prácticas investigativas y artísticas desde el performance, donde se utilizó el cuerpo como instrumento educativo para expresar voces y sentires de un colectivo de jóvenes de ciclo $\mathrm{V}$ del colegio Francisco de Paula Santander I.E.D.

La idea nace desde la iniciativa de dos docentes de la institución, quienes lograron identificar actitudes de intolerancia ante la diferencia, apatía por los ejercicios sociopolíticos de la participación y desconocimiento de la historia local. Como objetivo, esta tesis se propuso analizar la incidencia de las prácticas estéticas, políticas y éticas en la formación de subjetividades de los participantes del colectivo "Trinchera: Bosa, Arte, Saberes y Territorio".

Metodológicamente, Morales y Pineda (2017) desarrollaron un tipo de investigación cualitativa, ya que buscaron describir la realidad del contexto a partir de la reflexión, confrontación y producción suscitada en ejercicios individuales y colectivos, acciones que permitieron legitimar la apreciación y opinión de los sujetos desde la diferencia y la heterogeneidad como dimensiones intrínsecas que forman parte de la suma social. La investigación cualitativa permitió comprender la particularidad de los sujetos y las colectividades, analizando la realidad desde las experiencias e inferencias procedentes de sus significados, sentimientos y percepciones. Frente a la intención, se hizo necesario definir como tipo de investigación la Investigación Interpretativa entendida como un proceso flexible y dinámico donde el investigador tiene la posibilidad de recolectar la información como insumo para la consolidación de conclusiones. El trabajo se estructuró a través del estudio de casos. Entre las técnicas y los instrumentos, utilizaron el trabajo de campo, entrevistas, documentos escritos y performance.

Como resultados que arrojaron la sistematización del proyecto Trinchera: Bosa, Arte, Saberes y Territorio se puede decir que se inscribe, como una alternativa viva para la construcción de nuevos sentidos comunicativos, al introducir en este campo novedosas acciones que exigieron la renovación de concepciones de maestros y jóvenes, la inmersión en los contextos sociales y la creación de iniciativas para la construcción de una vida en común. Además, "Trinchera" agenció reconocimiento y posicionamiento en diversos ambientes institucionales, mediante la incursión en las plataformas tecnológicas; YouTube ha sido uno de los escenarios interactivos que dio acceso a la proyección de videos, entrevistas y reflexiones del acontecer, el desarrollo y avance de la propuesta. Otro resultado fue la concepción del operar desde el elemento performance como un acto político, pues en el ejercicio simbólico, el colectivo recreó el devenir de nuevas construcciones de una vida en común.

Ghiso (20II) en su artículo Sistematización. Un pensar el hacer, que se resiste a perder su autonomía, recoge las voces de varios educadores y educadoras frente a la percepción que se tiene de sistematizar. Tiene por objetivo rescatar algunas ideas claves que permitan contextualizar y problematizar los discursos que se vienen planteando sobre "Sistematización de prácticas sociales y educativas", y dejar abiertas algunas preguntas e inquietudes.

El autor inicia haciendo un cuestionamiento frente al problema de la instrumentación de gestión e información, aunque reconoce que no hay técnicas neutras. La contrariedad está en la incapacidad de resistir a un sistema de pensamiento, de información, de gestión y de acción impuesto por lógicas de poder capaces de promover e instaurar una mirada única sobre la realidad circundante. Es ahí donde las personas se olvidan de la necesidad de reflexionar críticamente frente a lo que se les propone, eligen y hacen, llegando al extremo de creer que eso es lo normal, racional, digno y ético.

Como resultados, el autor deja una reflexión frente a la experiencia y su sistematización ya que no solo son posibles en el lenguaje y la cultura, configuradores de los significados que los actores le otorgan a la práctica. Es decir, se debe producir conocimiento para la transformación y es en este proceso y contexto donde se originan cambios en la descripción, expresión, explicación, comprensión y valoración del quehacer. Otro resultado fue que, en el momento de generar y socializar los textos sobre la práctica, durante la sistematización, se pueden originar conflictos en torno a la experiencia realizada, porque las narrativas la rehacen, la desordenan, la reordenan y resignifican desde las perspectivas de los diversos sujetos involucrados en ella.

Dentro de los artículos, tesis, escritos y documentos relacionados con el proyecto Centro de Expresión Cultural, aparece un documento institucional producido por Fe y Alegría (s.f.) que nos muestra una breve descripción frente a la misión 
y visión del Centro de Expresión Cultural (CEC) en el periodo comprendido de 1988 al 2006. Tiene como objetivo dar a conocer la propuesta formativa como apuesta educativa y social que busca la transformación del ser y de la comunidad.

Este documento está divido en cuatro momentos. El primero donde se contextualiza al lector con la propuesta, a partir de elementos como: origen, ubicación, historia e intencionalidad del CEC. Un segundo en el cual el autor expone la propuesta pedagógica aterrizada a cuatro áreas de desarrollo en las cuales se pueden desarrollar procesos de empoderamiento y fortalecimiento de capacidades en los sujetos participantes. Dichas áreas (cognitiva, psicológica, económica y política) transversalizan el proyecto que desde el arte capacita, enseña y orienta habilidades y potencialidades de los niños y niñas. Un tercero en el cual Fe y Alegría (s.f.) exponen las cinco modalidades artísticas bajo las cuales el proyecto trabaja con la comunidad. Es así, como la música, la danza, las artes plásticas, el teatro y el deporte son los talleres ofrecidos que en esa época dan cuenta de una gran cantidad de la población de la Localidad quinta de Usme participando activamente de dichos procesos. Por último, el documento muestra los equipos de trabajo que hacen que la propuesta marche y sea ejecutable para toda la comunidad.

Como resultados Fe y Alegría deja un documento inicial que permite a sus lectores dar a conocer el proyecto Centro de Expresión Cultural como un espacio de aprendizajes y construcción de experiencias desde el arte como elemento central para la transformación social de la comunidad.

Dando continuidad a los textos relacionados con el proyecto aparece Barragán (20I I) en EI CEC y su influencia sobre mi hacer como artista Teología de la liberación, educación popular vida en comunidad en este escrito relata desde su experiencia, cómo el Centro de Expresión Cultural ha influenciado su vida personal y profesional haciendo de ella un cuestionamiento constante frente a sus prácticas comunitarias, pero también académicas. Como bien lo menciona Barragán (201 I) Exponer la historia propia sirve para encontrar lo particular, pero también lo común, lo que nos une, la construcción del reino implica una construcción de comunidad, una experiencia, que no se aprende únicamente desde el discurso, sino que aparece desde el hacer, y por supuesto desde la reflexión sobre el hacer. (p.5)

La reflexión constante sobre nuestras acciones hace que la experiencia misma se enriquezca, pero sobre todo nos convoque en espacios donde consideramos que el encuentro con los otros nos lleva a la construcción misma de nuestros saberes presentes en los lugares que cotidianamente habitamos y que muchas veces pasan desapercibido cuando nos negamos la posibilidad del diálogo con uno mismo y con los demás.

Para concluir con este relato, el autor cuenta lo que sucedió con él a nivel profesional y personal después de haber dejado el proyecto, y cómo este influenció en los procesos formativos de los que hizo parte después.

\section{Marco teórico}

Esta aproximación teórica se realiza como punto de partida para el proyecto. En este orden de ideas, se plantearán tres grandes conceptos, que servirán como herramientas teóricas iniciales. El primero es experiencia estética vista desde la relación entre hombre y entorno, cultura y comunidad. Como segundo concepto se encuentra el empoderamiento visto desde elementos como la participación y el agenciamiento humano. Por último, un tercer concepto de participación visto desde las distintas experiencias en ámbitos sociales.

\section{Experiencia estética}

Para Dewey (2008) el concepto de estética está directamente relacionado con aspectos psicológicos en donde el interior del individuo desde su mente y cuerpo, genera un criterio consciente e inconsciente para diferenciar qué es o no bello a partir de un sentimiento o imaginación, pero que en relación a la experiencia entendida como la relación directa hombre - naturaleza- hombre, se vuelve aquella cualidad que le da solidez al concepto, lo que Dewey (2008) llama experiencia estética.

Para tener una aproximación al concepto de experiencia estética que nos plantea el autor se debe tener claridad en dos elementos psicológicos que parten de aspectos individuales. Por un lado, el concepto de sentimiento, entendido como un escalón más arriba de la percepción. El segundo, el conocimiento, entendido como el manejo básico de las informaciones que surge como consecuencia del sentimiento. Cuando el hombre inicia su camino a partir de los sentimientos, se genera la contemplación desde la experiencia vivida, cuando el individuo entra en contacto con su entorno pasando por una serie de situaciones producto de su continua acción. Es ahí, según Dewey (2008) en donde como experiencia, empieza a surgir una interacción entre el organismo y el entorno. Dicha experiencia acaba cuando el individuo encuentra sentido a la situación vivida, justo en ese momento inicia otra, enlazándose así, múltiples cadenas de experiencias vividas.

Todo esto en relación a las múltiples experiencias compartidas en el CEC, muestran elementos fundamentales de la formación de un sujeto social capaz de reconocer desde aspectos individuales y colectivos, sentimientos y percepciones que le permiten construir conocimiento, pero sobre todo empoderarse del saber que vivencia en su comunidad, teniendo como punto de partida su propia experiencia en un espacio donde puede diversificar su saber a las generaciones que vienen detrás en búsqueda de aprendizajes grupales.

Por otro lado, Rancière (201 I), en su libro Aisthesis aborda este concepto desde diferentes corrientes artísticas como la literatura, el teatro, la danza, entre otros. El origen de la palabra estética viene del griego 'sensación', 'sensibilidad', e orden de ideas, la Aisthesis es un régimen de identificación del arte, es el modo mediante el cual se designan una variedad de producciones pertenecientes al arte. Pero esta designación se da por múltiples factores. Por un lado, se encuentran las condiciones materiales en las cuales se encuentran los lugares 
de exposición, las formas de circulación, de producción y de reproducción del objeto artístico. Por el otro lado, se incluye un factor de percepción, de emoción, de sensibilidad, a través del cual se clasifican estas producciones dentro de esta esfera.

El arte es un mundo aparte, y es capaz de acoger objetos e ideas que se oponen a la idea del "arte bello" precisamente porque el régimen de percepciones, sensaciones e interpretaciones se altera, se reconstituye y se transforma con el paso de la historia. Las nuevas emergencias artísticas obligan a modificar los paradigmas del arte y este se va redefiniendo con las mutaciones de nuestros tejidos sensibles.

La estética configura, y, en una relación recíproca y dependiente, se establece desde la cultura y la comunidad. En la escena, por ejemplo, Rancière (20II) no ve un simple lugar en donde se ven plasmados los ideales, sino que se trata de "una pequeña máquina óptica" que muestra las ideas y los pensamientos que establecen los lazos que unen las sensibilidades y las percepciones. Es a partir de la escena en donde se constituye y se encuentra la comunidad sensible e intelectual que hace pensable lo que él llama el "Régimen estético del arte". Es decir, las transformaciones no son individuales, la escena provee el espacio en que se entretejen opiniones que darán lugar a las metamorfosis en las lógicas de percepción, afección y pensamiento.

\section{Empoderamiento}

Para iniciar a hablar del término empoderamiento, es importante mencionar algunas premisas que autores como Freire (1980), Rowlands (1997), Rappaport (1977) entre otros, dieron a conocer y que permitieron darle paso a lo que hoy por hoy entendemos del concepto en sí. Por un lado, Freire (1980) desde la noción de "pedagogías liberadoras" abre camino en propuestas educativas a nivel mundial que pasan de situaciones de dependencia a compromisos por liberarse. Dichas situaciones se ven expuestas desde una mirada principalmente económica que afecta a los países Latinoamericanos, generando condiciones de imposición y dependencia sobre la población, ya que las posibilidades de progreso son mínimas haciendo que se mantenga la concepción de países subdesarrollados. Esta situación de subordinación de unos países sobre otros, evidencian intereses de sublevación y dominio en escenarios como lo cultural, lo social y lo político. Frente a esta situación Freire (1980) realiza una reflexión de la realidad económica en América Latina en la cual plantea que el fenómeno del subdesarrollo no tiene su razón en sí misma, sino que la razón está en el desarrollo. A partir de estas interpretaciones surgen ciertas tendencias pedagógicas denominadas pedagogías de la liberación. Estas se incorporaron en propuestas educativas en lugares del mundo donde la situación de dependencia impulsa el compromiso de transformación. Desde este concepto, a pesar de que no se menciona explícitamente el término de empoderamiento, sí se puede decir que se da comienzo a una serie de elementos que poco a poco apuntan a generar en los sujetos posiciones críticas en búsqueda de estrategias por cambiar su realidad.

Por otro lado, Rowlands (1997) propone tres dimensiones desde donde se puede concebir el concepto de empoderamiento: lo personal, lo colectivo y las relaciones cercanas. La primera, permite desarrollar confianza en sí mismo y en las capacidades individuales para superar la opresión. La segunda, lo colectivo, suma esfuerzos individuales para lograr mayor impacto en un fin común. Como tercera y última, el autor comenta las habilidades de negociación, comunicación, defensa de derechos y dignidad que se deben construir con los sujetos. A partir de lo que propone Rowlands (I997) deben existir espacios que muestren iniciativas individuales en donde progresivamente se vaya hacia un bien común, así las comunidades adquieren relevancia cuando sus habitantes son los que se apropian de las dinámicas y funcionamiento, más no la institución. En este punto se retoma la tercera dimensión de la que el autor nos habla y es el momento en el cual los procesos trascienden de los agentes ejecutantes de los proyectos, es decir, las instituciones que muchas veces llegan a las poblaciones haciendo efectivos los presupuestos, usando a las comunidades para decir que si se están haciendo cosas pero en la cual no existe ningún tipo de prolongación, aún más preocupante, no fomentan procesos de empoderamiento en donde sean los sujetos de dichos lugares los que den continuidad. Es por eso que se hace urgente generar espacios donde existan verdaderas apropiaciones y re significaciones del conocimiento y del saber.

Por último, Rappaport (1977) introduce el modelo empowerment el cual busca mejorar la calidad y bienestar de vida desde los recursos individuales, grupales y comunitarios. Esta propuesta concibe nuevos entornos en los que se pueden evidenciar mayor capacidad para controlar su propia vida. Rappaport (1977) los expone desde tres componentes básicos: La ciencia social, la acción política y el desarrollo del recurso. Esta primera busca la resolución de problemas sociales que exponen la distribución desigual de los recursos materiales y psicológicos. Para dicho fin, esta ciencia social requiere de una acción política que crea condiciones sociales permitiendo así, actuar sobre el entorno social, específicamente, poblaciones vulnerables que necesitan ser potenciadas a partir del desarrollo del recurso el cual no se limita a la prestación de un servicio, sino que genera nuevas formas de situarse y comprender la realidad. Con estos tres elementos, el autor busca desarrollar en los sujetos, organizaciones y comunidades conciencia sobre las necesidades de su entorno, generando así procesos de Desarrollo Humano Integral.

\section{Participación}

A lo largo del tiempo varios autores han hablado del concepto de participación desde escenarios en los cuales las comunidades describen experiencias en ámbitos sociales, ambientales, ciudadanos, entre otros. Stringer (1972) muestra tres versiones de participación a partir de elaboraciones personales que se construyen desde las vivencias. Por un lado "Tener parte de" cuando se aceptan los constructos de las organizaciones, instituciones y demás entidades en las cuales los usuarios pueden involucrarse pero que de alguna forma no se tiene en cuenta la posición de los participantes. "Tomar parte en" cuando los sujetos se ajustan a las dinámicas en las cuales están inmersos o bien imponen sus posiciones, pero que de alguna forma existe una adaptación de parte y parte. Por último "Ser parte de" cuando los proyectos elaborados se dan de forma integral, es decir, una relación comunicativa que garantiza la 
participación de los usuarios inmersos en las dinámicas de las organizaciones y así crear constructos colectivos en donde las dos partes participan. Con esto el autor plantea una relación interacción en la que los usuarios y las entidades exponen sus puntos de vista dándole validez a sus posiciones en donde aprenden y enseñan conocimientos que benefician al proyecto.

Cunill (199|) quien habla de la participación comunitaria como un estado de carácter netamente asistencial ya que involucra a la comunidad en la solución de problemas de su vida diaria. Claro está, que este tipo de participación a la que se refiere el autor, habla de los años 60 y 70 en donde se buscaba mejorar las condiciones de vida de la población, mientras que la participación comunitaria de hoy busca una relación directa con el estado en donde la comunidad tenga influencia en la construcción y práctica de la propuesta. Es entonces cuando se elaboran estrategias que tengan influencia a nivel político, cultural y social.

Para Torres (2006) históricamente han existido múltiples formas de participación a partir de movilizaciones, proyectos y estrategias de resistencia cotidiana en los movimientos sociales de América Latina. Estos elementos reflejan manifestaciones y expresiones que ilustran la capacidad de supervivencia y resistencia organizativa desde las voluntades y esfuerzos en la resolución de problemas y en la proyección de utopías. Para el autor, Bogotá ha sido el escenario de emergencia de múltiples experiencias protagonizadas por los pobladores populares que al versen enfrentados a condiciones precarias, se asocian para buscar solución a sus necesidades en busca de una interlocución con el estado y el impulso de sus iniciativas. Desde esta perspectiva la conformación de proyectos sociales y culturales parten de la necesidad por contribuir al mejoramiento y proyección de las comunidades, en este caso, hablo de la construcción del proyecto CEC que en manos de la Comunidad de las Hermanas Carmelitas de la Caridad Vedruna permitió la formación artística de varios niños y jóvenes de la Localidad. A partir de esta práctica y como lo menciona Torres (2006) se generan nuevas subjetividades y sentidos de pertenencia, ya que en el momento en que se reconoce la incidencia que estos tienen en las identidades personales, se está abordando una identidad colectiva, una construcción propia elaborada desde los mitos, símbolos, ritos, lenguajes y valores que le dan un sello único frente a otras asociaciones similares. Para el autor, la identidad no se configura tanto en el poseer una historia común y en participar de una ideología, sino en el hecho de mantener conversaciones recurrentes, propósitos y vínculos. Además de compartir costumbres, símbolos, valores y creencias que garantizan la continuidad de las acciones y la cohesión de sus miembros en torno a ellas. Mirándolo de ese modo, es importante mencionar que en el CEC el arte fue el eje articulador que permitió una asistencia constante de parte de la comunidad. Fue el espacio para la invención y la creación de múltiples propuestas en donde los sujetos colectivizaron sus saberes en pro de una necesidad común, la visibilización de experiencias cotidianas, problemáticas sociales y el aprendizaje de otros lenguajes. Pero, sobre todo, el encuentro constante de niños y jóvenes que pensaron en una sociedad diferente, más incluyente, colectivizada, pensada para todos.

Ortega y Torres (20II) nos hablan de reflexionar el que hacer educativo desde una construcción social direccionada a lo cultural y lo político, entendiendo la educación como una opción social alternativa necesaria para lograr transformaciones individuales y colectivas. Estos autores retoman los planteamientos de Lola Cendales quien refiere que todo proceso educativo es eminentemente ético- político ya que las prácticas pedagógicas deben dar cuenta de las relaciones con el contexto, buscando trabajar por la construcción de una nueva sociedad, sin exclusión en busca de posibilidades dignas para todos. Para esto es necesario llegar a los escenarios socioculturales donde existan procesos de trasformación de los sujetos desde sus realidades. Ahora bien, en este ejercicio por entender dichas prácticas, es necesario visibilizar la memoria de las comunidades desde ejercicios participativos y procesos de constitución de sujetos que configuren una nueva visión de la pedagogía la cual como lo dice Lola, adquiere vida desde la práctica cotidiana de maestros y educadores.

Con estas premisas, los espacios pensados en el CEC para la comunidad, más allá de la formación artística, generaron iniciativas de diálogos entre los participantes, diálogos que a su vez fueron construidos desde las experiencias vividas en el hogar, en el barrio, en el colegio y demás lugares habitados cotidianamente. Es desde estas cotidianidades que los sujetos elaboran y configuran sus discursos en donde progresivamente van solidificando una experiencia colectiva política y cultural ya que se convierte en un hábito reiterativo el asistir al taller, compartir con sus compañeros y encontrarse con diálogos comunes.

\section{Métodología}

La presente investigación se enmarca dentro del enfoque cualitativo. Este paradigma cuya vida es de tan solo unas décadas, más allá de los números y la búsqueda de patrones, se vale de procedimientos descriptivos para caracterizar y comprender un fenómeno social. En otras palabras, según Strauss y Corbin (2002), la investigación cualitativa busca llegar a descubrimientos que no se hallan exclusivamente a través de procedimientos estadísticos u otros medios de cuantificación. Esta perspectiva es empleada por investigadores en educación, sociología, psicología, estudios de comunicación, estudios culturales y otros tipos de estudios sociales que buscan evaluar el desarrollo normal de ciertos sucesos.

Así mismo, como se mencionó inicialmente, este enfoque, según Sampieri (20/4), no pretende generalizar ni encontrar patrones que permitan llevar los resultados a otros sujetos o poblaciones más amplias. De hecho, la indagación cualitativa no aspira a obtener necesariamente muestras representativas, sino que, como define Patton (20I I), los datos cualitativos describen detalladamente las situaciones, conductas o eventos observados. Se caracteriza porque a través de esta se estudia el vivir de la gente, su cotidianidad, sus experiencias, sus comportamientos, sus percepciones, emociones y sentimientos, el sujeto y la comunidad, así como los fenómenos culturales.

Ahora bien, teniendo en cuenta que el arte no solo es para contemplar, sino que es un acto y como tal hace parte de una interacción y construcción colectiva que incide en la cultura de un lugar, ya que de alguna manera está influenciando las 
formas de pensar y percibir el mundo de los sujetos que allí se encuentran, se puede decir que espacios como los del Centro de Expresión Cultural están encaminados en la construcción de percepciones, buscando otras formas de ver y sentir, esto de la mano con la necesidad que tienen las personas de hacer parte de ámbitos y territorios por los que se va pasando a lo largo de su vida. La mayoría de niños y jóvenes buscan un lugar diferente al de la casa y escuela para conocer personas contemporáneas, relacionarse y convivir en la construcción de aprendizajes colectivos donde ellos aporten desde su saber.

Esta investigación se realizó en el periodo de 1998 hasta el 2018 , debido a que desde ese año la mayoría de los participantes construyeron su proyecto de vida alrededor de todas las experiencias y los aprendizajes que vivieron en el CEC durante su infancia y adolescencia, es decir, esta población decidió empezar su búsqueda por llegar a otros espacios en los cuales enriquecieron su saber desde las prácticas académicas en carreras afines de artes, ciencias de la educación o ciencias humanas y proyectos comunitarios relacionados con procesos de formación infantil y juvenil. Actualmente el $80 \%$ de estos sujetos, desde su proyección laboral, hace parte de procesos comunitarios en educación artística.

La primera, es la entrevista a profundidad (Taylor y Bogdan, 1992) la cual se realizó a tres familias seleccionadas que reúnen características similares, ya que son habitantes de la localidad quinta de Usme con trayectoria de más de 25 años viviendo en el lugar y más de 10 Para ello, se hace uso de un paradigma cualitativo, ya que es a partir de las voces y experiencias de las familias participantes del CEC que se busca identificar y comprender el fenómeno en relación a sus contextos y sus propias historias de vida.

Son familias monoparentales, lideradas por madres cabeza de hogar, interesadas en ofrecer mejores oportunidades para sus familias, con una escolaridad inferior al bachillerato $y$, quienes participan del CEC recogiendo fondos para la ampliación y construcción del edificio donde funcionaría el bachillerato, los salones de artes y el teatro para la comunidad. Dichas actividades fueron bazares, coca colas bailables, bingos, rifas, entre otros.

La segunda generación se caracteriza por ser en su mayoría mujeres con hijos desde muy jóvenes, algunas cuentan con carreras profesionales, otras finalizaron el bachillerato y se dedicaron a laborar. Su participación inicia en los diferentes talleres que para esa época estaban activos en el CEC (deportes, música, artes plásticas, teatro, danzas, break dance, entre otros). Alterno a esto hacen parte del grupo de animadores conformado por adolescentes de los diferentes grupos cuyo objetivo es formarse como líderes de grupo y compartir su saber con niños y niñas de menor edad. Esto permitió que la construcción de su proyecto de vida tuviera una orientación hacia la enseñanza de las artes.

La tercera generación se encuentra actualmente escolarizada y en su tiempo libre asiste a los talleres de danza y música infantil y pre-juvenil. Es importante mencionar que esta generación llega al espacio a raíz de todo un legado familiar que reconoce el proyecto como un lugar de aprendizaje, formación en valores $y$ en habilidades, que les permita consolidar su personalidad, su carácter y su lectura del contexto a través del arte.

Como segunda técnica está el análisis visual (Acaso, 2009), en este ejercicio se propuso un análisis fotográfico que mostró los diferentes eventos llevados a cabo entre los años de 1998 a 2018. Dichos eventos están relacionados con presentaciones artísticas, asambleas juveniles, conversatorios, convivencias y foros locales. Debido a la gran cantidad de registro fotográfico existente en el CEC, se hizo necesario seleccionar algunas fotografías teniendo en cuenta tres criterios:

I) Fotografías asociadas a los integrantes de las tres familias entrevistadas. Es decir, no necesariamente debe aparecer en la imagen, pero si puede tener algún vínculo o relación con esta.

2) Fotografías que evidencien los diferentes eventos realizados con los niños y jóvenes pertenecientes a los grupos de música y danza del CEC, evidenciando así, los procesos internos trabajados con la población involucrada.

3) Fotografías que muestren los distintos tipos de población participante del proyecto (niños, jóvenes y adultos).

Por último, se realizó un taller pedagógico basado en el concepto de Ghiso (1997), que para este ejercicio de sistematización se desarrolló dentro de la muestra artística del año 2018, producto del trabajo de formación en los talleres de danza y música, del cual hicieron parte las tres familias vinculadas en este proceso. Dicho taller cuenta con tres momentos que recogen un dialogo intergeneracional en el cual cada integrante de las familias cumple un papel fundamental.

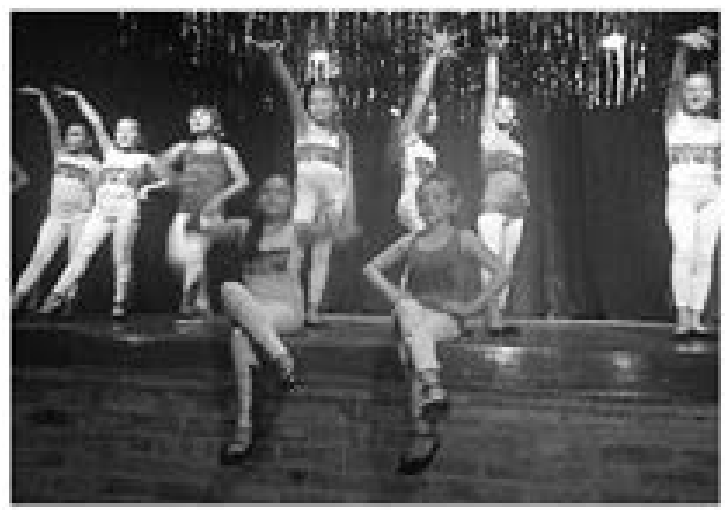

llustración I. Presentación de cierre de año 2017. Grupo "Estrellas del swing".

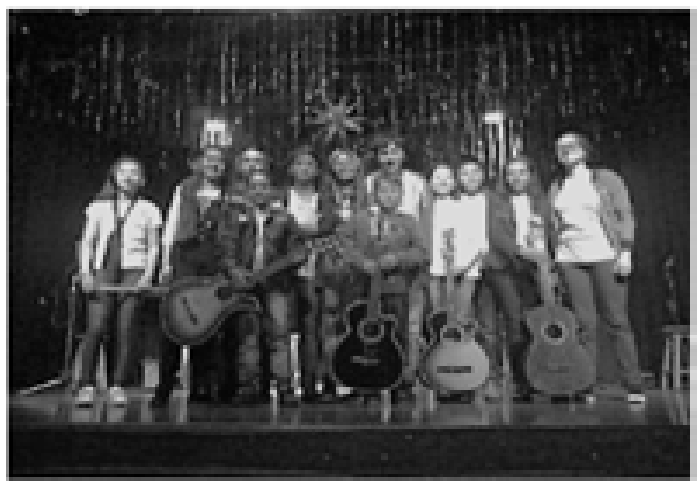

Ilustración 2. Grupo de música juvenil del año 2018. 


\section{Análisis y resultados}

A partir de la información recolectada a la luz de los objetivos propuestos para esta sistematización se identifican fundamentalmente algunas categorías emergentes entre los cuales se encuentran: empoderamiento, comunidad experiencia estética, formación humana, memoria histórica y saberes intergeneracionales. Desde el análisis de estas categorías se muestran los siguientes resultados:

I) EI CEC para muchos sujetos representó y representa la posibilidad de abrir nuevos caminos para ver y concebir el mundo desde otras ópticas generando cuestionamientos políticos, sociales y culturales que los sujetos a diario reconocen en sus contextos.

2) Es importante mencionar que a pesar de que en la localidad existen otras ofertas para la formación artística, el CEC mantiene sus dinámicas de trabajo desde experiencias individuales y colectivas en donde la comunidad conoce $y$ reconoce el proyecto como un lugar de formación infantil y juvenil.

3) El CEC es uno de los escenarios de participación infantil y juvenil que a través de los años ha formado líderes comunitarios que multiplican su saber en otros escenarios, dejando como legado en la comunidad un reconocimiento en la memoria individual y colectiva de todas las personas que han transitado por este lugar y que de alguna u otra manera han sido impactados por esta experiencia. Impacto, que se visibiliza desde los campos académicos, profesional y personal, donde los sujetos pueden poner en práctica, aquellos saberes y experiencias para mejorar sus condiciones de vida y del entorno del que hacen parte.

\section{Conclusiones}

Esta sistematización muestra las experiencias vividas en el Centro de Expresión Cultural de Santa Librada, proyecto de formación humana y artística, obteniendo algunas conclusiones elaboradas en dos líneas. Por una parte, se pretende reflexionar sobre los ejercicios de sistematización en espacios no formales que han posibilitado el reconocimiento de prácticas pedagógicas diversas en múltiples escenarios, por otro, dar cuenta de algunas reflexiones y aprendizajes adquiridos durante este proceso llevado a cabo, en donde la comunidad desde su participación da cuenta de la importancia de este proyecto en el sector.

En primer lugar, la sistematización de experiencias como ejercicio de producción desde la práctica es una acción que debe ir tomando fuerza y relevancia, para que los procesos de organización popular sirvan de reflexión y contribuyan a la construcción de saberes que no solamente se encuentran en los espacios institucionales, generando nuevas alternativas sociales y culturales para que el conocimiento sea un ejercicio de diversos saberes construidos desde acciones individuales y colectivas.

Los procesos de sistematización en escenarios comunitarios deben involucrar la voz de los sujetos, permitiendo que el ejercicio se enriquezca y le dé un verdadero reconocimiento a quienes hicieron posible el desarrollo de la experiencia. Dicha experiencia no solo recoge datos del pasado, sino que permite la construcción colectiva del conocimiento para que los sujetos se apropien de su experiencia, se reconozcan y empoderen, generando acciones sociales con proyección hacia futuro.

Para ello, fue vital el uso de técnicas como el análisis visual, las entrevistas y el taller pedagógico porque posibilitaron el encuentro con otros a través de la palabra, esto permitió la recuperación de la memoria histórica haciéndola visible y dándole un lugar en la comunidad. Las entrevistas permitieron hilar la historia del CEC en las voces de tres generaciones con experiencias, necesidades y aprendizajes distintos, mientras que el análisis visual evidenció los avances, cambios o retrocesos que se han dado en el espacio respecto a la formación, formas de participación y encuentro.

Por último, es importante sistematizar las prácticas pedagógicas en espacios formales y no formales para dar a conocer los procesos que se están dando a nivel educativo, social y cultural, así los aprendizajes se nutren y permiten ejercicios de retroalimentación constante del saber pedagógico.

En segundo lugar, frente a la sistematización del CEC se evidencia un amplio proceso artístico y humano presente en la localidad quinta de Usme, que ha beneficiado a centenares de niños, niñas y jóvenes en pro del descubrimiento y reconocimiento de habilidades que les permiten posicionarse en otros campos y escenarios sociales, culturales y académicos dentro y fuera del país relacionados con educación popular, trabajo comunitario y educación artística.

Por otra parte, es de resaltar el papel de la memoria histórica en este ejercicio de sistematización, ya que más allá de reconocer en el arte una alternativa de transformación a la realidad, permitió recordar y mantener vivo los orígenes del CEC, evitando que queden en el olvido esas tradiciones, ideas y aprendizajes que permitieron consolidar el proyecto, tal y como se le conoce, contribuyendo así a que las nuevas generaciones reconozcan lo que ya se ha hecho y a partir de allí propongan nuevas ideas, más acordes con el momento histórico y con las necesidades del contexto.

Teniendo en cuenta los momentos de crisis y altibajos por los que el CEC ha atravesado y a pesar de los esfuerzos por mantenerlo vivo, el espacio requiere de una financiación que permita atender a las demandas de formación del contexto en el que se encuentra ahora. De lo contrario, en poco tiempo el proyecto culminará. Esto debido a que poco a poco el espacio se ha reducido cediéndole gran parte de la planta física a otros proyectos que arriendan el espacio para la ejecución de sus proyectos.

Esta experiencia de sistematización fue de gran importancia para mí como investigadora ya que me permitió aprender, compartir y construir conocimiento con la comunidad con la que crecí. Considero que el ejercicio adquiere más relevancia cuando los investigadores conocen el proceso desde su propia experiencia. Es decir, ser parte de este, hace que se 
amplíen y se enriquezcan los conceptos al tener no solo la visión de investigadora, sino de participante.

Siendo el Centro de Expresión Cultural un espacio de formación que me permitió construir mi proyecto de vida, rescato la posibilidad de haber dado a conocer esta experiencia, de alguna forma como una retribución y agradecimiento por todo lo que me enseñó y formó en mí. 


\section{Referencias bibliográficas}

Acaso, M. (2009) El lenguaje visual. Barcelona, España: Paidos.

Alfaro, L. \& Sura, C. (2007). Teatro comunitario como proceso de transformación social. (Tesis pregrado, Universidad Tecnológica Metropolitana).Recuperado de centroderecursos.alboan.org/ebooks/0000/0680/6_UTE_TEA.pdf.

Barragán, J. (20I I) El CEC y su influencia sobre mi hacer como artista Teología de la liberación, educación popular vida en comunidad. Recuperado el 3 de Noviembre de 201 I de http://mde.org.co/mde I I/wp-content/uploads/sites/3/20 I I/I I/Jaime-EBarrag\%C3\%AIn-El-CEC-.pdf.

Cunill, N. (199I) Participación ciudadana, dilemas y perspectivas para la democratización de los estados Latinoamericanos. Recuperado en 2015 www.probidadenchile.cl/wp/cunill-nuria-participacion-ciudadana/.

Dewey, J. (2008). El arte como experiencia. Claramonte, J. (Trad.). España: Paidós.

Fe y Alegría (s.f.) Centro de Expresión Cultural (CEC) Santa Librada 1988 - 2006. Bogotá, Colombia. Recuperado de www. feyalegria.org/es/...no.../centro-expresion-cultural-santa-librada-fya-colombia

Freire, P. (1980). El mensaje de Paulo Freire. Teoría y práctica de la liberación. Madrid, España: Marsiega.

Ghiso, A. (1997). El taller en procesos investigativos interactivos. Recuperado de virtual.funlam.edu.co/.../acercamientos_ al_taller_recurso_propio_unidad_3.891.pdf

Ghiso, A. (20II) Sistematización. Un pensar el hacer, que se resiste a perder su autonomía. Revista Decisio (28), 3 - 8. Recuperado de www.cepalforja.org/sistem/documentos/decisio28_saberl.pdf.

Lozano, J., Alonso, P., Puche, J. (2003) Centro de Expresión Cultural Fe y Alegría Santa Librada (CEC) Una experiencia de Fe y Alegría en Colombia. Bogotá. Recuperado de

www.feyalegria.org/es/...no.../centro-expresion-cultural-santa-librada-fya-colombia

Morales L. y Ortiz E. (2017). Trinchera: Bosa, Arte, Saberes y Territorio: una alternativa agonística de hacer escuela (Tesis de maestría, Universidad Distrital Francisco José de Caldas). Recuperado de repository.udistrital.edu.co/bitstream/II349/.../ MoralesPelaezLeidyCatherine2017.pdf.

Ortega, P y Torres, A. (20II) Lola Cendales González, entre trayectos y proyectos en la educación popular. Revista Colombiana de Educación (6I). I5-22. Recuperado www.scielo.org.co/pdf/rcde/n6I/n6la I5.pdf.

Rancière, J. (20I I). Aisthesis, Escenas del régimen estético del arte. París, Francia: Éditions Galilée.

Rowlands, J. (1997) Empoderamiento en mujeres rurales en Honduras: un modelo para el desarrollo. En León, M. (Coord.), Poder y empoderamiento de las mujeres (pp. 213-245). Bogotá: Tercer Mundo Editores S.A.

Taylor S. y Bogdan R. Introducción a los métodos cualitativos en investigación. La búsqueda de los significados. Ed. Paidós, España, 1992-Pág-100 - | 32. Recuperado de https://www.onsc.gub.uy/enap/.../Entrevista_en_profundidad_Taylo_y_Bogdan.pdf

Torres. A (2006) Organizaciones populares, construcción de identidad y acción política. Revista Latinoamericana de ciencias sociales, Niñez y Juventud (4), 3- 18. Recuperado de biblioteca.clacso.edu.ar/Colombia/alianza-cinde-umz/.../art.AlfonsoTorresC.pdf. 